\title{
First glimpse of the soft x-ray induced excited spin-state trapping effect dynamics on spin cross-over molecules
}

V. Davesne, M. Gruber, T. Miyamachi, V. Da Costa, S. Boukari, F. Scheurer, L. Joly, P. Ohresser, E. Otero, F. Choueikani, A. B. Gaspar, J. A. Real, W. Wulfhekel, M. Bowen, and E. Beaurepaire

Citation: The Journal of Chemical Physics 139, 074708 (2013); doi: 10.1063/1.4818603

View online: https://doi.org/10.1063/1.4818603

View Table of Contents: http://aip.scitation.org/toc/jcp/139/7

Published by the American Institute of Physics

\section{Articles you may be interested in}

Spin crossover in $\mathrm{Fe}$ (phen) 2 (NCS) 2 complexes on metallic surfaces

The Journal of Chemical Physics 146, 092312 (2017); 10.1063/1.4973511

Hysteresis and change of transition temperature in thin films of $\mathrm{Fe}\left\{\left[\mathrm{Me}{ }_{2} \mathrm{Pyrz}\right]_{3} \mathrm{BH}\right\}_{2}$, a new sublimable spincrossover molecule

The Journal of Chemical Physics 142, 194702 (2015); 10.1063/1.4921309

Study of molecular spin-crossover complex Fe(phen) $)_{2}(\mathrm{NCS})_{2}$ thin films

Applied Physics Letters 95, 043303 (2009); 10.1063/1.3192355

Electrical properties and non-volatile memory effect of the $\left[\mathrm{Fe}\left(\mathrm{HB}(\mathrm{pz})_{3}\right)_{2}\right]$ spin crossover complex integrated in a microelectrode device

Applied Physics Letters 99, 053307 (2011); 10.1063/1.3616147

Activation of coherent lattice phonon following ultrafast molecular spin-state photo-switching: A molecule-tolattice energy transfer

Structural Dynamics 3, 023605 (2016); 10.1063/1.4936290

Microelectromechanical systems integrating molecular spin crossover actuators

Applied Physics Letters 109, 061903 (2016); 10.1063/1.4960766

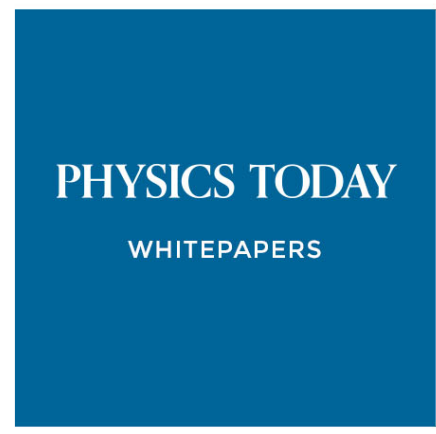

ADVANCED LIGHT CURE ADHESIVES

Take a closer look at what these environmentally friendly adhesive systems can do

\section{READ NOW}

PRESENTED BY Q MASTERBOND 


\title{
First glimpse of the soft x-ray induced excited spin-state trapping effect dynamics on spin cross-over molecules
}

\author{
V. Davesne, ${ }^{1,2, a)}$ M. Gruber, ${ }^{1,2}$ T. Miyamachi, ${ }^{3, b)}$ V. Da Costa, ${ }^{1}$ S. Boukari, ${ }^{1}$ F. Scheurer, ${ }^{1}$ \\ L. Joly, ${ }^{1}$ P. Ohresser, ${ }^{4}$ E. Otero, ${ }^{4}$ F. Choueikani, ${ }^{4}$ A. B. Gaspar, ${ }^{5}$ J. A. Real, ${ }^{5}$ \\ W. Wulfhekel, ${ }^{2}$ M. Bowen, ${ }^{1}$ and E. Beaurepaire ${ }^{1}$ \\ ${ }^{1}$ Institut de Physique et de Chimie des Matériaux de Strasbourg, Université de Strasbourg, CNRS UMR 7504 \\ 23 rue du Loess, 67034 Strasbourg, France \\ ${ }^{2}$ Physikalisches Institut, Karlsruhe Institut of Technology, Wolfgang-Gaede-Str. 1, 76131 Karlsruhe, Germany \\ ${ }^{3}$ Institute for Solid State Physics, University of Tokyo, 5-1-5 Kashiwanoha, Kashiwashi, \\ Chiba 277-8581, Japan \\ ${ }^{4}$ Synchrotron SOLEIL, L'Orme des Merisiers, Saint-Aubin, BP48, 91192 Gif-sur-Yvette, France \\ ${ }^{5}$ Institut of Molecular Science, Universitat de València, Edifici de Instituts de Paterna, \\ Apartat de Correus 22085, 46071 València, Spain
}

(Received 27 May 2013; accepted 1 August 2013; published online 21 August 2013)

The dynamics of the soft $\mathrm{x}$-ray induced excited spin state trapping (SOXIESST) effect of $\mathrm{Fe}(\text { phen })_{2}(\mathrm{NCS})_{2}$ (Fe-phen) powder have been investigated by x-ray absorption spectroscopy (XAS) using the total electron yield method, in a wide temperature range. The low-spin (LS) state is excited into the metastable high-spin (HS) state at a rate that depends on the intensity of the x-ray illumination it receives, and both the temperature and the intensity of the x-ray illumination will affect the maximum HS proportion that is reached. We find that the SOXIESST HS spin state transforms back to the LS state at a rate that is similar to that found for the light induced excited spin state trapping (LIESST) effect. We show that it is possible to use the SOXIESST effect in combination with the LIESST effect to investigate the influence of cooperative behavior on the dynamics of both effects. To investigate the impact of molecular cooperativity, we compare our results on Fe-phen with those obtained for $\mathrm{Fe}\left\{\left[\mathrm{Me}_{2} \mathrm{Pyrz}\right]_{3} \mathrm{BH}\right\}_{2}$ (Fe-pyrz) powder, which exhibits a similar thermal transition temperature but with a hysteresis. We find that, while the time constant of the dynamic is identical for both molecules, the SOXIESST effect is less efficient at exciting the HS state in Fe-pyrz than in Fe-phen. (C) 2013 AIP Publishing LLC. [http://dx.doi.org/10.1063/1.4818603]

\section{INTRODUCTION}

Spin cross-over (SCO) molecules have garnered increasing scientific attention due to their interesting potentialities as intrinsic switches. ${ }^{1,2}$ Indeed, those complexes can undergo a change in their spin state by applying various external stimuli so as to alter the ligand field around the magnetic site. $\mathrm{Fe}(\mathrm{phen})_{2}(\mathrm{NCS})_{2}$ (phen $=1,10$-phenanthroline, see Fig. 1(a); denoted Fe-phen hereafter) is a well-studied SCO complex ${ }^{3}$ that can change from a $S=0$, low-spin (LS) state to a $S=2$, high-spin (HS) state by increasing the temperature above $176 \mathrm{~K},{ }^{3}$ by illumination at low temperature (LIESST) ${ }^{4-6}$ or through pressure. ${ }^{7}$ We have shown that the SCO property of Fe-phen can withstand physical vapor deposition. ${ }^{8}$ This in turn allowed us to study single-molecule, SCO-based memristors, ${ }^{9}$ in which the electric current govern the molecule's spin state, as an elegant and robust path toward memristive spintronics. ${ }^{10}$

The spin transition has been studied by a wide range of techniques, including Mössbauer spectroscopy, magnetic susceptibility and reflectivity, ${ }^{11}$ and optical absorption, for which

\footnotetext{
a) Author to whom correspondence should be addressed. Electronic mail: vincent.davesne@ipcms.unistra.fr

b) This research was performed while T. Miyamachi was at the Physikalisches Institut, Karlsruhe, Germany.
}

good reviews ${ }^{12,13}$ exist, but $\mathrm{x}$-ray absorption spectroscopy (XAS) can, advantageously, shed light on the electronic structures of both states, which makes it particularly interesting for fundamental investigations, ${ }^{14}$ especially on thin films and isolated molecules. ${ }^{9,15,16}$ Furthermore, it can not only be used as an investigation technique, but can also trigger the LS-to-HS transition at low temperatures (SOXIESST effect) as demonstrated by Collison et al. ${ }^{17}$ (not to be confused with the similar effect given by hard $\mathrm{x}$-ray excitation ${ }^{18}$ ).

Until now, it has been suspected that the SOXIESST effect resembles the light-induced excited spin state trapping (LIESST), but this similarity has not been investigated in detail. The relaxation of LIESST-generated HS into LS has already been investigated by XAS. ${ }^{19}$ As we will detail hereafter the influence of the SOXIESST effect should not be overlooked under $60 \mathrm{~K}$ as it may lead to a time-dependence of the LS-to-HS transition within this temperature range. Since the LIESST process has been already nearly entirely elucidated, ${ }^{20}$ we aim to draw the attention of the scientific community to the similarities and differences between both effects.

We find that the two effects follow similar dynamics and are complementary one to another, which suggests that, as with the LIESST effect, ${ }^{11}$ cooperativity plays an important role in the SOXIESST effect. To test 
(a)

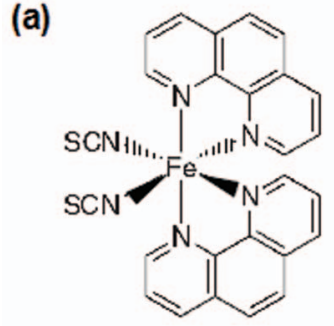

(b)

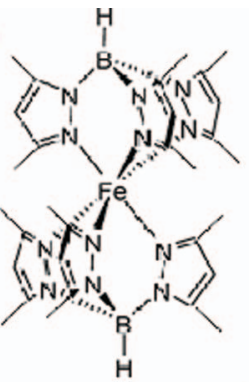

(c)

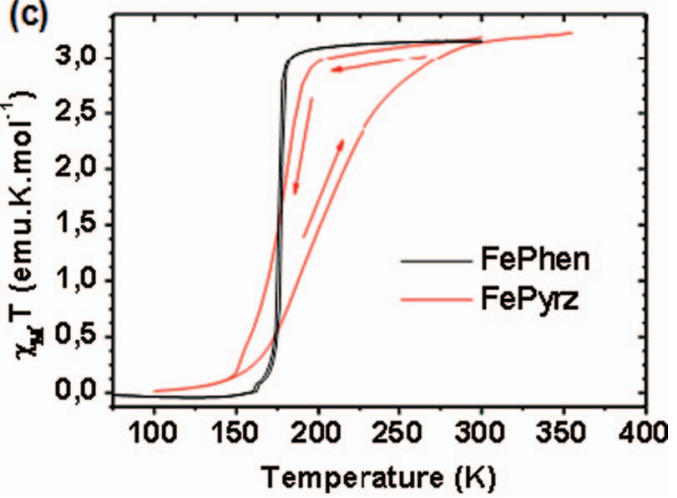

FIG. 1. Schematic view of (a) $\mathrm{Fe}(\text { phen })_{2}(\mathrm{NCS})_{2}$ and (b) $\mathrm{Fe}\left\{\left[\mathrm{Me}_{2} \mathrm{Pyrz}\right]_{3} \mathrm{BH}\right\}_{2}$. (c) Temperature-dependent measurement of Fe-pyrz susceptibility measured by SQUID. Arrows indicate the temperature sweep direction.

this tenet, we compare with another molecular species, $\mathrm{Fe}\left\{\left[\mathrm{Me}_{2} \mathrm{Pyrz}\right]_{3} \mathrm{BH}\right\}_{2}$ (Fe-pyrz, see Fig. 1(b)), whose spin transition is accompanied by a thermal hysteresis. We find that, while the time constants are similar, the x-ray excitation seems less efficient. Overall, since the effect has been at times overlooked, ${ }^{19}$ our results indisputably confirm that the SOXIESST effect exists. In turn, we provide guidelines and practical considerations on the possible effects one might come across while studying SCO molecules using XAS.

\section{METHODS}

\section{A. Molecules}

The $\mathrm{Fe}(\text { phen })_{2}(\mathrm{NCS})_{2}$ powder used in this study was synthesized by G. Rogez using a standard technique, ${ }^{21}$ while the $\mathrm{Fe}\left\{\left[\mathrm{Me}_{2} \mathrm{Pyrz}\right]_{3} \mathrm{BH}\right\}_{2}$ powder was synthesized by A. B. Gaspar from the group of Professor J. A. Real, using an improved synthesis compared to that reported in the litterature: ${ }^{22}$ the coumpound was synthetized under $\mathrm{Ar}$ atmosphere by adding dropwise $2 \mathrm{mmol}$ of the salt $\mathrm{K}\left[\mathrm{HB}\left(3,5-\left(\mathrm{CH}_{3}\right)_{2} \mathrm{pz}\right)_{3}\right]$ dissolved in methanol $(15 \mathrm{ml})$ to a methanolic solution containing $1 \mathrm{mmol}$ of $\mathrm{Fe}\left(\mathrm{BF}_{4}\right) 2 \cdot 6 \mathrm{H}_{2} \mathrm{O}(5 \mathrm{ml})$. After complete addition of the $\mathrm{K}\left[\mathrm{HB}\left(3,5-\left(\mathrm{CH}_{3}\right)_{2} \mathrm{pz}\right)_{3}\right]_{2}$ solution a white precipitate appears. The precipitate was washed with methanol and dried under vacuum, for a yield of $60 \%$. For x-ray absorption measurements, powders were pressed onto a tantalum plate that was fixed onto a copper sample holder, which was then mounted in the cryostat.

\section{B. X-ray absorption spectroscopy: Data acquisition and analysis}

XAS is a powerful technique to investigate $\mathrm{SCO}$, since it is element-specific and depends on the transitions between core and valence electrons. This makes it possible to witness the electronic difference between the two spin states. XAS experiments were carried out on beamline DEIMOS at the SOLEIL synchrotron. ${ }^{23}$ XAS spectra were acquired in total electron yield mode. Until now, this technique was too slow to witness the dynamics of the SOXIESST effect, but thanks to recent experimental improvements on beamline DEIMOS, the time required to acquire one spectrum dropped from 20 to $1 \mathrm{~min}$, thereby enabling slow dynamics studies.

Since our experimental tool, namely XAS, can alter the HS proportion through the SOXIESST effect, particular care was taken in implementing the correct experimental methodology. Where noted, studies of the SOXIESST dynamics were obtained by acquiring a series of XAS spectra at a stabilized temperature, oftentimes only over the $\mathrm{L}_{3}$ edge so as to gain in temporal resolution. The subsequent measurement of the HS proportion is then determined using the first XAS spectrum of this series, i.e., within 1 min of sample illumination with $\mathrm{x}$-rays. This inferred HS proportion is called the virgin $H S$ proportion. The time stamp of each subsequent measurement corresponds to the midpoint of the measurement. This allowed us to investigate the SOXIESST dynamics (see Figs. 3(a) and 3(b)). We label the stabilized HS proportion due to SOXIESST the SOXIESST HS proportion, determined using the last XAS spectrum of the series. Subsequent measurements of the SOXIESST HS proportion were then achieved by first resetting the sample's HS proportion by heating the sample to $80-100 \mathrm{~K}$ while blocking the $\mathrm{x}$-ray beam, then stabilizing the subsequent temperature. As another experimental protocol, we first measured a SOXIESST HS proportion at $4 \mathrm{~K}$, and then increased temperature to $60 \mathrm{~K}$ while performing XAS measurements. The inferred value of this HS proportion is called the SOXIESST without (w/o) reset HS proportion. Unless noted, samples were not exposed to visible light before or during XAS measurements so as to avoid the LIESST effect. When the additional impact of LIESST was investigated, visible light excitation was achieved using standard white LEDs. This is denoted as the SOXIESST + LIESST $H S$ proportion. We have also explored, starting from $4 \mathrm{~K}$ and while maintaining $\mathrm{x}$-ray and LED illumination, the temperature dependence of the HS proportion, which is then labeled the SOXIESST w/o reset + LIESST HS proportion. Practical examples of these denominations can be found in Figs. 3(a) and $3(b)$.

Finally, as previously reported, ${ }^{17,19}$ we also observe the degradation of the Fe-phen molecule over time (the so-called soft x-ray photochemistry), which is manifested by the freezing of a LS state, probably through the removal of one of the ligand groups. But, at an estimated photon density of $6.4 \times 10^{9} \mathrm{ph} / \mathrm{s} / \mathrm{mm}^{2}$, this process is sufficiently slow for Fephen: we estimate that the rate of this process is under $3 \%$ of the sample under the $\mathrm{x}$-ray beam $/ \mathrm{h}$. A similar behavior was observed for Fe-pyrz, with a rate of approximately $11 \%$ of the sample/h at $6.1 \times 10^{8} \mathrm{ph} / \mathrm{s} / \mathrm{mm}^{2}$. As expected, at low 
illumination, both molecules suffer far less degradation. Therefore, we recommend low XAS illumination parameters for the measurement of Fe(II) SCO compounds, as they still allow the easy recognition of the spin states while avoiding undesirable side effects.

This allowed us, over the course of several runs, to notably study the impact of x-ray photon density on the SOXIESST effect. The first run utilized an estimated photon density $\mathrm{I}_{0}=6.1 \times 10^{8} \mathrm{ph} / \mathrm{s} / \mathrm{mm}^{2}$ in single bunch filling mode: a single bunch of $20 \mathrm{~mA}$ is circulating in the synchrotron ring, illuminating the sample at a frequency of $0.847 \mathrm{MHz}$; for the second run $\mathrm{I}=6.4 \times 10^{9} \mathrm{ph} / \mathrm{s} / \mathrm{mm}^{2} \approx 10 \mathrm{I}_{0}$; and for the third run $\mathrm{I}=1.2 \times 10^{10} \mathrm{ph} / \mathrm{s} / \mathrm{mm}^{2} \approx 20 \mathrm{I}_{0}$ both in hybrid filling mode: in this mode, $3 / 4$ of the ring is filled with 312 bunches of $1.36 \mathrm{~mA}$, with the last quarter of one single $5 \mathrm{~mA}$ bunch, illuminating the sample at a frequency of $352.2 \mathrm{MHz}$. The diaphragm openings were kept constant throughout the three series of measurements.

\section{RESULTS}

Referring to Fig. 2(a), the Fe- $\mathrm{L}_{2,3}$ edge spectra exhibit a clear temperature variation between two extremal spectra obtained at $280 \mathrm{~K}$ or $300 \mathrm{~K}$, and $100 \mathrm{~K}$. These spectra have been ascertained, in the literature ${ }^{14,17,19,24}$ but also in our previous study, ${ }^{9}$ to belong to the Fe-phen pure HS and LS states, respectively. For a given XAS spectrum, we may extract the HS
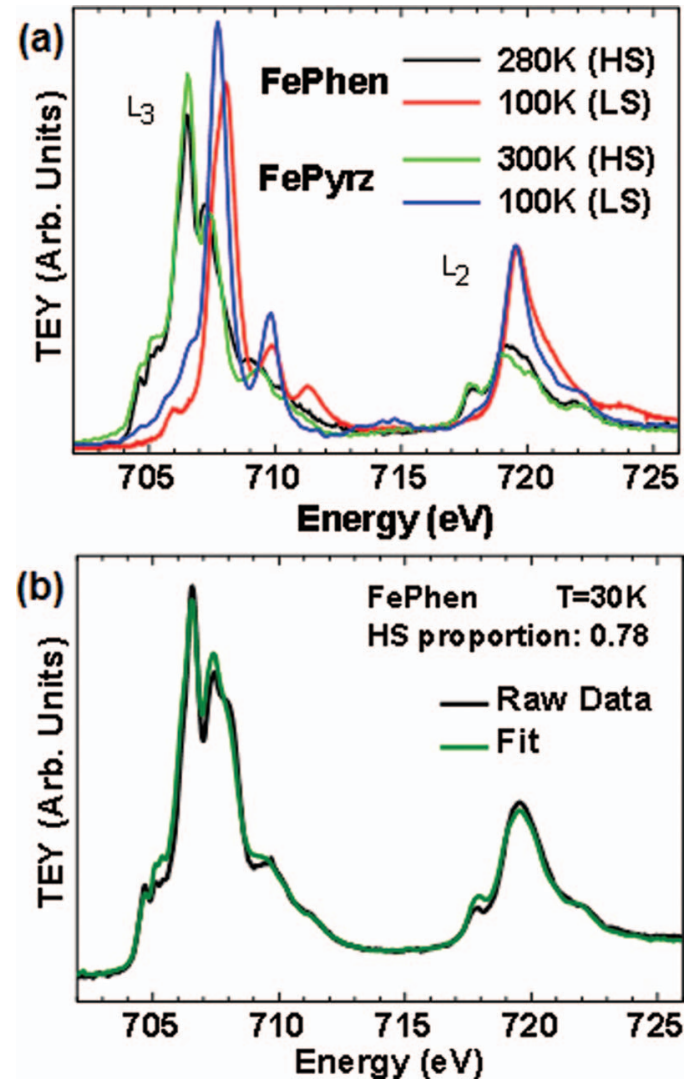

FIG. 2. (a) XAS reference spectra, obtained using photon intensity $\mathrm{I}_{0}$, of the HS and LS states of Fe-phen and Fe-pyrz powders. (b) Raw Fe-phen XAS spectrum measured at $\mathrm{T}=30 \mathrm{~K}$ and $\mathrm{I}=10 \mathrm{I}_{0}$ and fit to the raw XAS spectrum using a combination of the HS/LS reference spectra (78\% HS, 22\% LS).

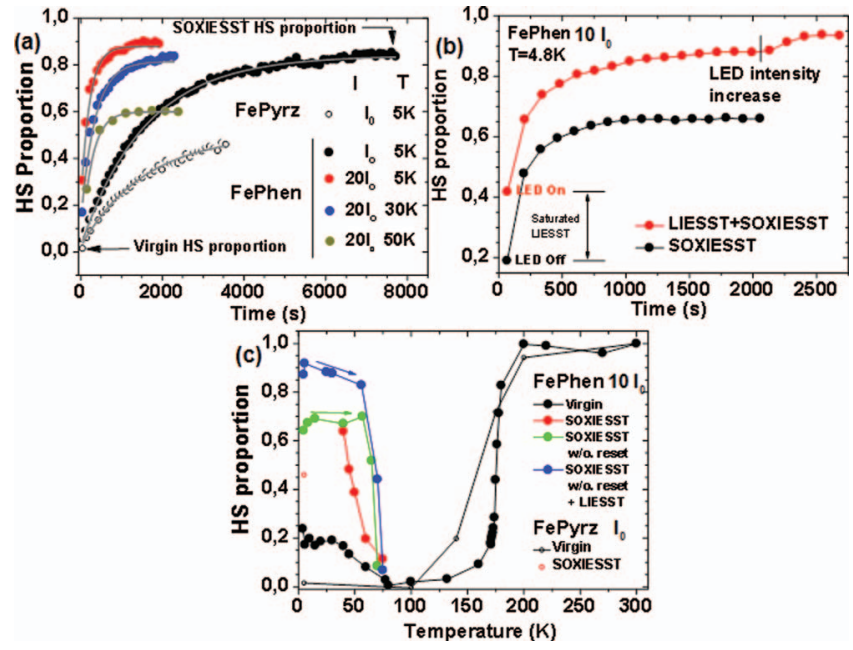

FIG. 3. (a) Examples of the Fe-phen HS proportion dynamics that illustrate the impact of temperature and x-ray intensity. A Fe-pyrz dataset is also plotted for comparison. Lines represent fits using Eq. (1). For each SOXIESST dynamics spectrum, the stabilized value of the HS proportion was used to plot the temperature dependence of the SOXIESST HS proportion shown in Fig. 3(c). (b) SCO dynamics promoted by SOXIESST alone and LIESST + SOXIESST for $10 \mathrm{I}_{0}$. The light-excited data-set was recorded by first illuminating the sample with light for $30 \mathrm{~min}$ to achieve a LIESST-saturated HS proportion, then recording the SOXIESST dynamics spectrum. At the mark, the LED intensity was increased to achieve a LIESST-induced increase in the HS proportion. (c) Temperature dependence of the Fe-phen HS proportion, obtained for $10 \mathrm{I}_{0}$, for the virgin HS proportion (black); for the SOXIESST HS proportion (red, lower temperature points follow the green curve); for the SOXIESST w/o reset HS proportion obtained while increasing the temperature from $4 \mathrm{~K}$ under steady $\mathrm{x}$-ray illumination (green); and for the SOXIESST w/o reset + LIESST HS proportion obtained while increasing the temperature from $4 \mathrm{~K}$ under steady $\mathrm{x}$-ray and white light illumination (blue). A complementary subset of data, obtained using intensity $\mathrm{I}_{0}$, is plotted for Fe-pyrz using open symbols.

proportion by fitting the spectrum using a linear combination of these two reference spectra. A representative fit is shown in Fig. 2(b).

For a given temperature, we have studied the temporal evolution of the HS proportion. Examples of this extensive study are shown in Figs. 3(a) and 3(b). Comparing with previous results, ${ }^{11}$ despite the different photon flux, we observe that the process occurs in the same time frame as the LIESST. Indeed, both the SOXIESST and the LIESST reach saturation within hundreds to thousands of seconds. We also note that the SOXIESST-generated transition is not complete using intensity $10 \mathrm{I}_{0}$ since the LIESST effect, whether applied before or once the SOXIESST effect saturates, leads to a higher HS proportion (see Fig. 3(b)).

We plot in Fig. 3(c) the temperature dependence of the HS proportion. Notably, we observe a hysteresis between the SOXIESST HS proportion (red) and either the SOXIESST HS w/o reset proportion (green) or the SOXIESST w/o reset + LIESST HS proportion (blue). This appears to be a kinetic effect since the LIESST HS (metastable) $\rightarrow$ LS relaxation speed is very slow ${ }^{2,6}$ at those temperatures. All measurement protocols yield the same SOXIESST HS proportion at $\mathrm{T} \sim 60 \mathrm{~K}$. 


\section{DISCUSSION}

Contrary to previous studies ${ }^{17}$ in which a complete transition was achieved, we observe only a partial HS transition. The HS fraction reached upon SOXIESST with $10 \mathrm{I}_{0}$ light intensity can be increased by applying visible-UV light excitation as illustrated in Figures 3(b) (red) and 3(c) (blue). However, we suppose that this is due to a weaker x-ray illumination in our experimental conditions $\left(6.4 \times 10^{9}\right.$ photons $\mathrm{s}^{-1} \mathrm{~mm}^{-2}$ in this first case, compared with $10^{11}$ in Collison et al. ${ }^{17}$ ). This hypothesis is supported by our results at higher $\mathrm{x}$-ray intensity $20 \mathrm{I}_{0}$, for which the conversion is nearly complete (see Fig. 3(a)). We observe a hysteresis between the SOXIESST HS proportion and SOXIESST w/o reset proportion, as has similarly been observed for the LIESST effect. ${ }^{25}$

The previous study suggested that the SOXIESST and LIESST mechanisms should be similar: ${ }^{17}$ a LS state transitions to an excited state, then relaxes into either a LS or a metastable HS state. In turn, the HS state relaxes slowly to the LS state through intersystem crossing (ISC). ${ }^{4,26}$ When fitting a given experimental spectrum with reference HS and LS spectra so as to extract the HS proportion, we do not notice any anomalous deviation from the noise present in the remainder spectrum. This suggests that a possible excited transitional state relaxes at least within the $60 \mathrm{~s}$ duration of our XAS spectrum. According to the literature, ${ }^{11}$ the LIESST excitation and relaxation dynamics are self-accelerated due to cooperative effects, which leads to nonlinear dynamics. In our case, we simplify the model by disregarding the excited state and the cooperative effect and by just using the LS-to-HS conversion and the subsequent relaxation, with their respective rate constants:

$$
\text { LS } \stackrel{\mathrm{k} 1}{\rightarrow} \mathrm{HS}, \quad \mathrm{HS} \stackrel{\mathrm{k} 2}{\rightarrow} \text { LS }
$$

This leads to the following equation of evolution of the HS proportion:

$$
p_{H S}=\frac{-k_{1}}{k_{1}+k_{2}} \times e^{-\left(k_{1}+k_{2}\right) \times t}+\frac{k_{1}}{k_{1}+k_{2}} .
$$

This yields give $1 /\left(\mathrm{k}_{1}+\mathrm{k}_{2}\right)$ as the time constant and $\mathrm{k}_{1} /\left(\mathrm{k}_{1}+\mathrm{k}_{2}\right)$ as the saturation.

As a note, our experiments seem to indicate that the SOXIESST effect is not resonant. Indeed, we observe that the energy of the x-ray illumination and the HS proportion evolution are not correlated: the HS proportion dynamics are the same if we measure the subsequent spectra over $L_{3}$ and $L_{2}$ or only $\mathrm{L}_{3}$. Besides, a possible reverse-SOXIESST phenomenon may also exist, in analogy to the reverse-LIESST. ${ }^{27}$ Therefore, since we cannot identify precisely the beginning of the excitation, we allow within the fitting procedure a time offset so as to grant some freedom to temporally position the initial point of the SOXIESST process and we position the timestamp for each spectrum at the middle of the time window.

At first, we ascertain that $\mathrm{k}_{1}$ seems to be independent of the temperature above approximately $10 \mathrm{~K}$. This is in agreement with LIESST experiments, for which $\mathrm{k}_{1}$ is considered to mostly depend on the intensity and the probability of conversion. ${ }^{11}$ We thus fit the curves using a global $\mathrm{k}_{1}$

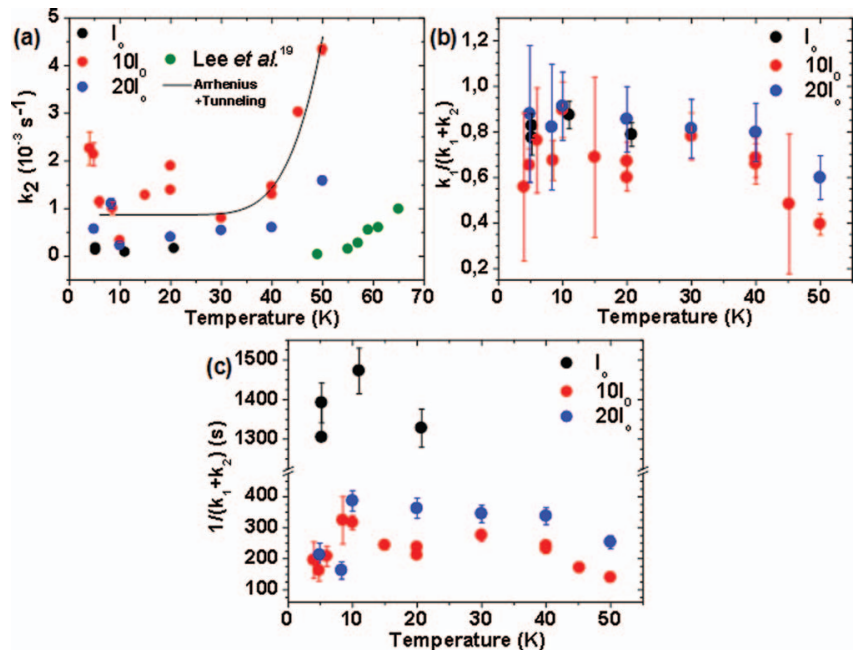

FIG. 4. Temperature dependence of the fitted values of (a) the HS $\rightarrow$ LS relaxation rate constant, with experimental values of Lee et al. ${ }^{19}$ and a fit of the $10 \mathrm{I}_{0}$ data points based on Eq. (2), (b) the saturation, $\mathrm{k}_{1} /\left(\mathrm{k}_{1}+\mathrm{k}_{2}\right)$, and (c) the time constant $1 /\left(k_{1}+k_{2}\right)$ of the SOXIESST effect for Fe-phen powder, for every SOXIESST dynamics and every intensity.

for all the SOXIESST dynamics with the same x-ray intensity. We find that $k_{1}$ for $I_{0}$ is $5.9 \times 10^{-4} \mathrm{~s}^{-1}$; for $10 \mathrm{I}_{0}$ is $2.8 \times 10^{-3} \mathrm{~s}^{-1}$; and for $20 \mathrm{I}_{0}$ is $3.0 \times 10^{-3} \mathrm{~s}^{-1}$.

The relaxation parameter $\mathrm{k}_{2}$, which corresponds to the relaxation of the metastable HS state into the LS state, has been already investigated in the case of the LIESST effect for several spin transition molecules. ${ }^{26}$ Experimental values of $\mathrm{k}_{2}$ resulting from LIESST determined by Lee et al. ${ }^{19}$ are plotted in Fig. 4(a) alongside our results for comparison. However, the actual HS $\rightarrow$ LS relaxation follows the equations for an ISC process, ${ }^{26}$ rather than the more common Arrhenius law. Above $30 \mathrm{~K},{ }^{13}$ we should include in the law a cooperative term that depends on the HS proportion: ${ }^{25} \mathrm{k}_{2}\left(\mathrm{~T}, \mathrm{P}_{\mathrm{HS}}\right)$ $=\mathrm{k}_{2}(\mathrm{~T}, 0) \times \exp \left(-\alpha(\mathrm{T}) \times \mathrm{P}_{\mathrm{HS}}\right), \alpha$ being proportional to the inverse of the temperature. This term is also present in the excitation. This could explain the dispersion in our results, since a slight HS proportion before illumination can induce drastic changes in the $\mathrm{k}_{2}$. Second, the ISC process exhibits a tunneling regime that makes it diverge from an Arrhenius model at low temperatures. In essence, some relaxation still occurs below $30 \mathrm{~K} .{ }^{13}$ To illustrate this discrepancy, we plot as a line in Fig. 4(a) a fit to the $10 \mathrm{I}_{0}$ data-set corresponding to the following modified Arrhenius equation:

$$
k 2=A \times e^{\left(\frac{-E a}{k_{B} \times T}\right)}+\lim _{T \Rightarrow 0} k 2(T) .
$$

Nevertheless, our results are in qualitative agreement with those from the previous LIESST dynamics experiments. ${ }^{19,26}$ Notably, we observe a stable $\mathrm{k}_{2}$ from $4 \mathrm{~K}$ to $40 \mathrm{~K},{ }^{26}$ and an exponential increase above $40 \mathrm{~K}$. We find $\mathrm{E}_{\mathrm{a}}=32 \pm 17 \mathrm{meV}$, compared to $61 \pm 9 \mathrm{meV}$ for the LIESST-excited relaxation in the litterature. ${ }^{19}$ Similarly, the relaxation rate is controlled mostly by the preexponential factor, as $\mathrm{A}=6.4$ for our results compared to $\mathrm{A}=57$ in Ref. 19.

For $\mathrm{T}<10 \mathrm{~K}$, we observe an apparent decreasing trend in the saturation (Fig. 4(a)), as well as a decrease in the time constant (Fig. 4(b)). A decrease of the magnetic moment (linked 
TABLE I. Fit parameters for Fe-pyrz and Fe-phen acquired using two x-ray intensities.

\begin{tabular}{ccccccc}
\hline \hline \multicolumn{2}{c}{ Intensity } & Temperature (K) & $\mathrm{k}_{1}\left(\mathrm{~s}^{-1}\right)$ & $\mathrm{k}_{2}\left(\mathrm{~s}^{-1}\right)$ & Time constant (s) & Saturation \\
\hline $\mathrm{I}_{0}$ & Fe-pyrz & 5 & $3.2 \times 10^{-4}$ & $3.2 \times 10^{-4}$ & 1560 & 0.50 \\
& Fe-phen & 5.2 & $5.9 \times 10^{-4}$ & $1.2 \times 10^{-4}$ & 1390 & 0.83 \\
$20 \mathrm{I}_{0}$ & Fe-pyrz & 11 & $1.4 \times 10^{-3}$ & $1.1 \times 10^{-3}$ & 395 & 0.56 \\
& Fe-phen & 10 & $3.0 \times 10^{-3}$ & $2.3 \times 10^{-4}$ & 386 & 0.91 \\
\hline \hline
\end{tabular}

to the HS proportion in the sample) has already been noted for the LIESST effect using magnetic measurements for various SCO molecules, ${ }^{28}$ but it has been attributed to various measurement artifacts. However, some of the proposed factors, such as the opacity of the sample preventing light penetration, the zero-field splitting, and antiferromagnetic coupling in the case of dinuclear compounds, cannot result from the XAS technique. Despite this, we still observe a similar effect. Therefore, we suggest a new explanation for the behavior: this could reflect a decrease in the effective $\mathrm{k}_{1}$, which is quasi-constant between $\mathrm{T}=10 \mathrm{~K}$ and $\mathrm{T}=50 \mathrm{~K}$. This could reflect a reduced molecular mobility, which impairs the structural change associated with the spin transition. Essentially, low-temperatures "freeze" the given spin state and impair the switch. On the other hand, the molecules located at the grain boundaries or interfaces may remain more mobile, which results for $\mathrm{T}<10 \mathrm{~K}$ in a "faster" transition and in a decrease in saturation. Our model does not account for this behavior, which would explain the anomalous increase in $\mathrm{k}_{2}$ and decrease in the time constant at temperatures under $10 \mathrm{~K}$.

Our extensive dataset appears to show an inconsistent progression toward higher SOXIESST HS proportion with X-ray intensity (compare panels (a) and (b) of Fig. 3). At present, we surmise that this might reflect spin transition dynamics induced by the synchrotron source itself. Indeed, the $I_{0}$ dataset was acquired in single bunch filling mode, while the other two datasets were acquired in hybrid mode. Since the single bunch filling occurs by bursts of $20 \mathrm{~mA}$ at $0.847 \mathrm{MHz}$, whereas the hybrid filling occurs by bursts of $1.36 \mathrm{~mA}$ at $352.2 \mathrm{MHz}$, such a difference might act upon the rate of conversion of a transitory state of the lifetime typically in the nanosecond regime. ${ }^{9,13}$ This would mean that in the synchrotron's hybrid mode, after a first photon bunch excites part of the sample the sample still exhibits a remaining transitory state when the next photon bunch illuminates the sample. Subsequent measurements beyond the scope of this work may clarify this point.

Figure 3(b) reveals that the LIESST and SOXIESST effects may be combined to achieve a higher HS proportion. This means that it is possible to incrementally deploy the LIESST (SOXIESST) effect and then, starting from a steady state, to study the dynamics of the SOXIESST (LIESST) effect. Our investigation involves the XAS technique, but one can imagine using the $\mathrm{X}$-ray source exclusively to irradiate while deploying another technique, such as magnetic susceptibility, to measure the HS proportion without altering it. In our case, at $\mathrm{T}=4.8 \mathrm{~K}$ and after renormalisation on the same quantity of matter, $\mathrm{k}_{2}=7.9 \times 10^{-4} \mathrm{~s}^{-1}$ for the sample that was pre-illuminated with white light, while $\mathrm{k}_{2}=2.0 \times 10^{-3} \mathrm{~s}^{-1}$ for the virgin sample. This suggests that the SCO dynamics are faster when part of the sample is already in the $\mathrm{HS}$ state at a given temperature, in agreement with the cooperativity processes that underscore how the SCO process is presently understood. ${ }^{11}$

As a test of how cooperativity may impact the SCO dynamics, we now compare our results on Fe-phen with those obtained on Fe-pyrz. As seen in Fig. 1(c), and in contrast to Fe-phen, Fe-pyrz exhibits a thermal hysteresis in its thermal spin transition. Yet the HS $\rightarrow$ LS transition is thermally sharper than the LS $\rightarrow$ HS transition. Since the transition sharpness reflects the strength of the intermolecular cooperativity that takes part in the transition, ${ }^{12}$ this suggests that the $\mathrm{HS} \rightarrow$ LS transition involves stronger intermolecular cooperativity than the LS $\rightarrow$ HS transition, for reasons that are yet unclear and outside the scope of this study. One possible origin lies with structural differences between both states. ${ }^{29}$ As seen in Fig. 2(a), the XAS spectra for Fe-pyrz are quite similar to those found for Fe-phen. Since both molecules share the same octahedral arrangement (including the nitrogen "cage"), this was to be expected.

The time constants for Fe-pyrz are very similar to those for Fe-phen, as exhibited in Table I. Yet, for the same intensity $\mathrm{I}_{0}$ and at $\mathrm{T}=5 \mathrm{~K}$, the SOXIESST HS proportion achieved by Fe-pyrz $(\sim 0.4$ at $3500 \mathrm{~s})$ is lower than that for Fe-phen ( $\sim 0.8$ at $3500 \mathrm{~s})$. This shows that Fe-pyrz requires greater $\mathrm{x}$-ray photon intensity than Fe-phen in order to convert. Comparing both spectra, we notice that a peak at $711 \mathrm{eV}$ is absent from the Fe-pyrz spectrum. This peak is believed to be related to the hybridization between the $\mathrm{Fe}$ (II) $3 \mathrm{~d}$ orbitals and the NCS ligands. ${ }^{22}$ While we have already ascertained that the SOXIESST effect is non-resonant (thus putting aside the "direct" influence of the $711 \mathrm{eV}$ absorption band on the mechanism), this suggest that metalligand charge transfer plays an important role in the SOXIESST effect, like in the LIESST. ${ }^{20}$ Taken together, these results suggest that, while the excitation process is the same for both molecules, compared to Fe-phen, the illumination of Fe-pyrz might less efficiently induce the LS $\rightarrow$ HS transition and, conversely, the HS $\rightarrow$ LS relaxation of Fe-pyrz might be more efficient. This would be in agreement with the more strongly cooperative $\mathrm{HS} \rightarrow \mathrm{LS}$, and more weakly cooperative LS $\rightarrow \mathrm{HS}$, transitions observed for Fe-pyrz (see Fig. 1(c)). This could result from a crystallographic phase transition, defect creation, or from an intrinsic lowcooperative LS form.

\section{CONCLUSION}

We have confirmed the existence of the SOXIESST effect using two different Fe(II) complexes. Beyond lifting doubts 
on the existence of the effect raised by the community, ${ }^{19}$ our results show that one should take into account its presence at low temperatures (under $60 \mathrm{~K}$ ). However, as we have established, the intensity of the illumination is crucially linked to the amplitude of the effect, and we postulate that weak illumination could result in an apparent absence of the effect, or a very weak influence as was observed recently. ${ }^{15}$ We have also ascertained the usefulness of the XAS technique in total electron yield detection mode toward investigating the dynamics of relatively slow processes such as X-ray excitation of a SCO molecule. While it was not possible to observe excited states for the SOXIESST effect, the progressive transformation of one spin state into another is clearly visible. Variations in inter-molecular cooperativity seem to affect the amplitude of the SOXIESST effect as well, but the time constants are left unchanged. We have shown that it is possible to combine two techniques to be used not only to generate a determined proportion of HS but also to investigate the dynamics of the transition, thereby enabling further studies of cooperativity between $\mathrm{SCO}$ molecules during the spin transition process.

\section{ACKNOWLEDGMENTS}

We thank G. Rogez for Fe-phen synthesis and J. Arabski for technical help prior to the experiments. We thank the SOLEIL staff for technical assistance and insightful discussions. We acknowledge funding from FrancoGerman University, from the Deutsche Forschungsgemeinschaft (DFG) within the Center for Functional Nanostructures (CFN) and the Baden-Württemberg Stiftung in the framework of the Kompetenznetz für Funktionale Nanostrukturen (KFN). We also thank Frontiers of Research in Chemistry and the Labex NIE for funding.

${ }^{1}$ J.-F. Létard, P. Guionneau, and L. Goux-Capes, Top. Curr. Chem. 235, 221 (2004).

${ }^{2}$ M. Marchivie, P. Guionneau, J. A. K. Howard, G. Chastanet, J.-F. Létard, A. E. Goeta, and D. Chasseau, J. Am. Chem. Soc. 124, 194 (2002).

${ }^{3}$ W. A. Baker, Jr. and H. M. Bobonich, Inorg. Chem. 3, 1184 (1964).

${ }^{4}$ S. Decurtins, P. Gutlich, K. M. Hasselbach, A. Hauser, and H. Spiering, Inorg. Chem. 24, 2174 (1985).

${ }^{5}$ R. Herber and L. M. Casson, Inorg. Chem. 25, 847 (1986).
${ }^{6}$ K. Kato, M. Takata, Y. Moritomo, A. Nakamoto, and N. Kojima, Appl. Phys. Lett. 90, 201902 (2007).

${ }^{7}$ A. Bousseksou, G. Molnár, J.-P. Tuchagues, N. Menéndez, É. Codjovi, and F. Varret, C. R. Chim. 6, 329 (2003).

${ }^{8}$ S. Shi, G. Schmerber, J. Arabski, J.-B. Beaufrand, D. J. Kim, S. Boukari, M. Bowen, N. T. Kemp, N. Viart, G. Rogez, E. Beaurepaire, H. Aubriet, J. Petersen, C. Becker, and D. Ruch, Appl. Phys. Lett. 95, 043303 (2009).

${ }^{9}$ T. Miyamachi, M. Gruber, V. Davesne, M. Bowen, S. Boukari, L. Joly, F. Scheurer, G. Rogez, T. K. Yamada, P. Ohresser, E. Beaurepaire, and W. Wulfhekel, Nat. Commun. 3, 938 (2012).

${ }^{10}$ M. Bowen, J.-L. Maurice, A. Barthélémy, P. Prod'homme, E. Jacquet, J.-P. Contour, D. Imhoff, and C. Colliex, Appl. Phys. Lett. 89, 103517 (2006).

${ }^{11}$ C. Enachescu, H. Constant-Machado, E. Codjovi, J. Linares, K. Boukheddaden, and F. Varret, J. Phys. Chem. Solids 62, 1409 (2001).

${ }^{12} \mathrm{P}$. Gütlich and H. A. Goodwin, Top. Curr. Chem. 233, 1 (2004).

${ }^{13}$ J. A. Real, A. B. Gaspar, and M. C. Muñoz, Dalton Trans. 2005, 2062.

${ }^{14}$ C. Cartier dit Moulin, P. Rudolf, A. M. Flank, and C. T. Chen, J. Phys. Chem. 96, 6196 (1992).

${ }^{15}$ B. Warner, J. C. Oberg, T. G. Gill, F. El Hallak, C. F. Hirjibehedin, M. Serri, S. Heutz, M.-A. Arrio, P. Sainctavit, M. Mannini, G. Poneti, R. Sessoli, and P. Rosa, J. Phys. Chem. Lett. 4, 1546 (2013).

${ }^{16}$ M. Bernien, D. Wiedemann, C. F. Hermanns, A. Krüger, D. Rolf, W. Kroener, P. Müller, A. Grohmann, and W. Kuch, J. Phys. Chem. Lett. 3, 3431 (2012).

${ }^{17}$ D. Collison, C. D. Garner, C. M. McGrath, J. F. W. Mosselmans, M. D. Roper, J. M. W. Seddon, E. Sinn, and N. A. Young, J. Chem. Soc. Dalton Trans. 1997, 4371.

${ }^{18}$ G. Vankó, F. Renz, G. Molnár, T. Neisius, and S. Kárpáti, Angew. Chem., Int. Ed. 46, 5306 (2007).

${ }^{19}$ J.-J. Lee, H. Sheu, C.-R. Lee, J.-M. Chen, J.-F. Lee, C.-C. Wang, C.-H. Huang, and Y. Wang, J. Am. Chem. Soc. 122, 5742 (2000).

${ }^{20}$ A. Cannizzo, C. J. Milne, C. Consani, W. Gawelda, C. Bressler, F. van Mourik, and M. Chergui, Coord. Chem. Rev. 254, 2677 (2010).

${ }^{21}$ K. Akabori, H. Matsuo, and Y. Yamamoto, J. Inorg. Nucl. Chem. 35, 2679 (1973).

${ }^{22}$ J. P. Jesson, J. Chem. Phys. 48, 2058 (1968).

${ }^{23}$ P. Ohresser, E. Otero, F. Choueikani, S. Stanescu, F. Deschamps, L. Ibis, T. Moreno, F. Polack, B. Lagarde, F. Marteau, F. Scheurer, L. Joly, J.-P. Kappler, B. Muller, and P. Sainctavit, J. Phys.: Conf. Ser. 425, 212007 (2013).

${ }^{24}$ V. Briois, C. C. dit Moulin, P. Sainctavit, C. Brouder, and A.-M. Flank, J. Am. Chem. Soc. 117, 1019 (1995).

${ }^{25}$ A. Desaix, O. Roubeau, J. Jeftic, J. G. Haasnoot, K. Boukheddaden, E. Codjovi, J. Linarès, M. Noguès, and F. Varret, Eur. Phys. J. B 6, 183 (1998).

${ }^{26}$ A. Hauser, P. Adler, S. Deisenroth, P. Gütlich, C. Hennen, H. Spiering, and A. Vef, Hyperfine Interact. 90, 77 (1994).

${ }^{27}$ P. Gütlich, Y. Garcia, and H. A. Goodwin, Chem. Soc. Rev. 29, 419 (2000).

${ }^{28}$ J.-F. Létard, L. Capes, G. Chastanet, N. Moliner, S. Létard, J.-A. Real, and O. Kahn, Chem. Phys. Lett. 313, 115 (1999).

${ }^{29}$ L. Capes, J.-F. Létard, and O. Kahn, Chem.-Eur. J. 6, 2246 (2000). 